\title{
A RARE CASE REPORT OF ILEAL DUPLICATION IN A YOUNG ADULT MALE
}

\author{
Arunabha Sinha1, Syed Nasiruddin², Balabhim Joshi3 ${ }^{3}$, Kirthana ${ }^{4}$ \\ ${ }^{1}$ Associate Professor, Department of Surgery, MVJ Medical College. \\ ${ }^{2}$ Postgraduate, Department of Surgery, MVJ Medical College. \\ ${ }^{3}$ Postgraduate, Department of Surgery, MVJ Medical College. \\ ${ }^{4}$ Postgraduate, Department of Surgery, MVJ Medical College.
}

\section{ABSTRACT}

\section{BACKGROUND}

Ileal duplication is a rare congenital abnormality that can present in any portion of the GI tract and it is observed in one out of 25000 deliveries. Most of the adult intestinal duplications are asymptomatic and remain undiagnosed for years until onset of complications. It is rarely associated with volvulus. Ileal duplication can be classified according to the shape as cystic (noted in more than 80\%) or tubular. Intestinal duplications may have an acute presentation or chronic complaints. In our case report, the patient presented with pain in abdomen with vomiting and melena. CT scan showed midgut volvulus secondary to malrotation. Intraoperatively, it shows ileal loops adhered to each other close to terminal ileum and hence proceeded with right hemicolectomy. Histopathologically, it was confirmed to be ileal duplication with gastric heterotopia.

\section{KEYWORDS}

Congenital Malformations, Ileal Duplication, Volvulus, Right Hemicolectomy.

HOW TO CITE THIS ARTICLE: Sinha A, Nasiruddin S, Joshi B, et al. A rare case report of ileal duplication in a young adult male. J. Evolution Med. Dent. Sci. 2016;5(89):6657-6660, DOI: 10.14260/Jemds/2016/1505

\section{BACKGROUND}

Alimentary tract duplication is a rare congenital abnormality that can present in any portion of the GI tract.[1]

It can arise from either the foregut (33\%), midgut (56\%) or the hindgut $(11 \%)$ and is more common in males.

Intestinal duplication is rarely reported in adulthood and often remains undiagnosed until onset of complications.[2] Furthermore, intestinal duplication of small bowel has been rarely associated with malrotation. [3]

It can present as vague abdominal pain, distension with or without complications like obstruction, bleeding, perforation or malignancy.

Diagnosis of ileal duplication, especially in small intestine, may be difficult attributed to inability to visualise with standard endoscopic investigation. So radiographic study such as ultrasonography and CT scan play an important role in establishing the correct diagnosis and guide in appropriate management.[4]

\section{CASE REPORT}

A 24-year-old male presented with history of pain abdomen since 2 weeks, which was intermittent and colicky. He also had 2 episodes of vomiting per day since 2 weeks, which was blood tinged on occasions. He also gave history of passage of dark coloured stools with no associated fresh bleed. No history of significant loss of appetite or weight loss. No history of fever noted.

Past history of similar episodes of intermittent pain since childhood. However, worsening of symptoms noted over the

Financial or Other, Competing Interest: None.

Submission 01-10-2016, Peer Review 27-10-2016,

Acceptance 02-11-2016, Published 07-11-2016.

Corresponding Author:

Dr. Arunabha Sinha,

Flat No. D-0 Shilpitha Crystal Apt,

$6^{\text {th }}$ Cross Kaggadasapura

Bengaluru-560093.

E-mail:arunabha_sinha@yahoo.com

DOI: $10.14260 /$ jemds $/ 2016 / 1505$ past 5 years, which aggravated over the past 2 weeks. He gives history of symptomatic treatment for the pain over the years with temporary relief. On general examination, he was found to be moderately built and nourished, conscious and oriented, afebrile and vitals were stable. Abdomen examination showed mild diffuse tenderness (more in the lower abdomen). No guarding or rigidity noted. Bowel sounds were exaggerated. Other systemic examination was normal.

\section{Investigations}

1. Routine investigations (complete blood counts, liver profile and renal profile, serum electrolytes, urine analysis) was within normal limits.

2. Chest x-ray PA view: Within normal limits.

3. Plain CT abdomen and pelvis: Volvulus at the level of umbilicus associated with dilatation of distal jejunal and ileal loops with air fluid levels were noted. Most dilated part of the ileum measuring about $4.5 \mathrm{cms}$.

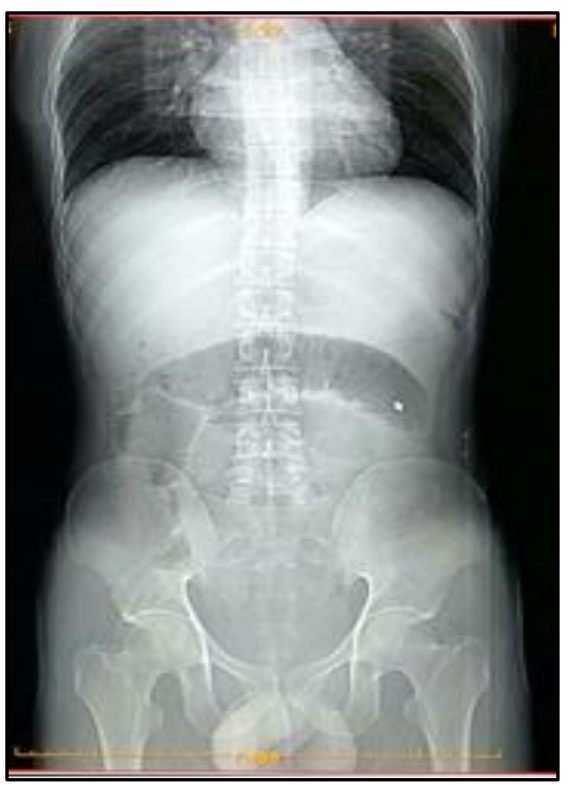

Figure 1. CT Scan Abdomen \&

Pelvis (Coronal View) 


\section{Preoperative Period}

On admission, patient was kept nil orally and given symptomatic treatment. He responded well to the same. On day 3, after admission he was started on liquid diet and on day 7 on soft diet. Once the patient was symptomatically better, patient was planned for elective exploratory laparotomy.

\section{Intraoperative Pictures}

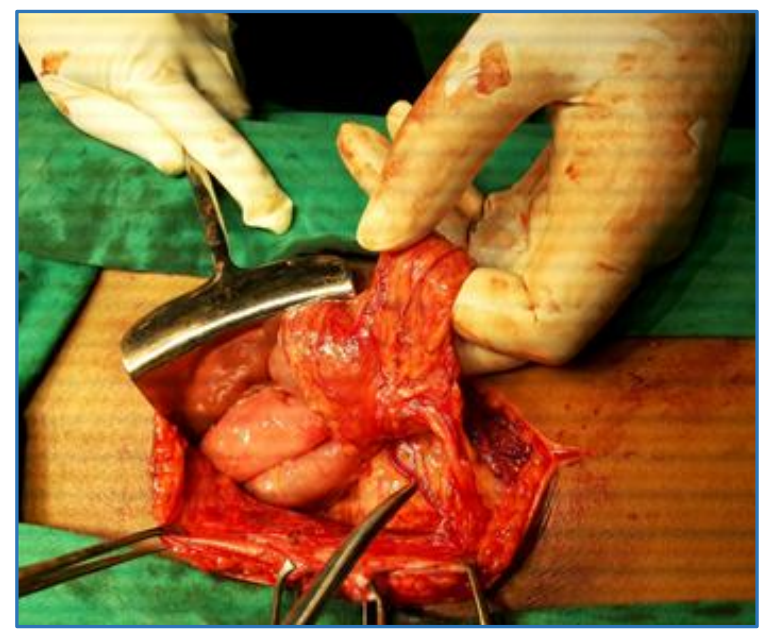

Figure 2. Intraoperative Image showing Falciform Ligament from the Umbilicus attached to the Mesentery
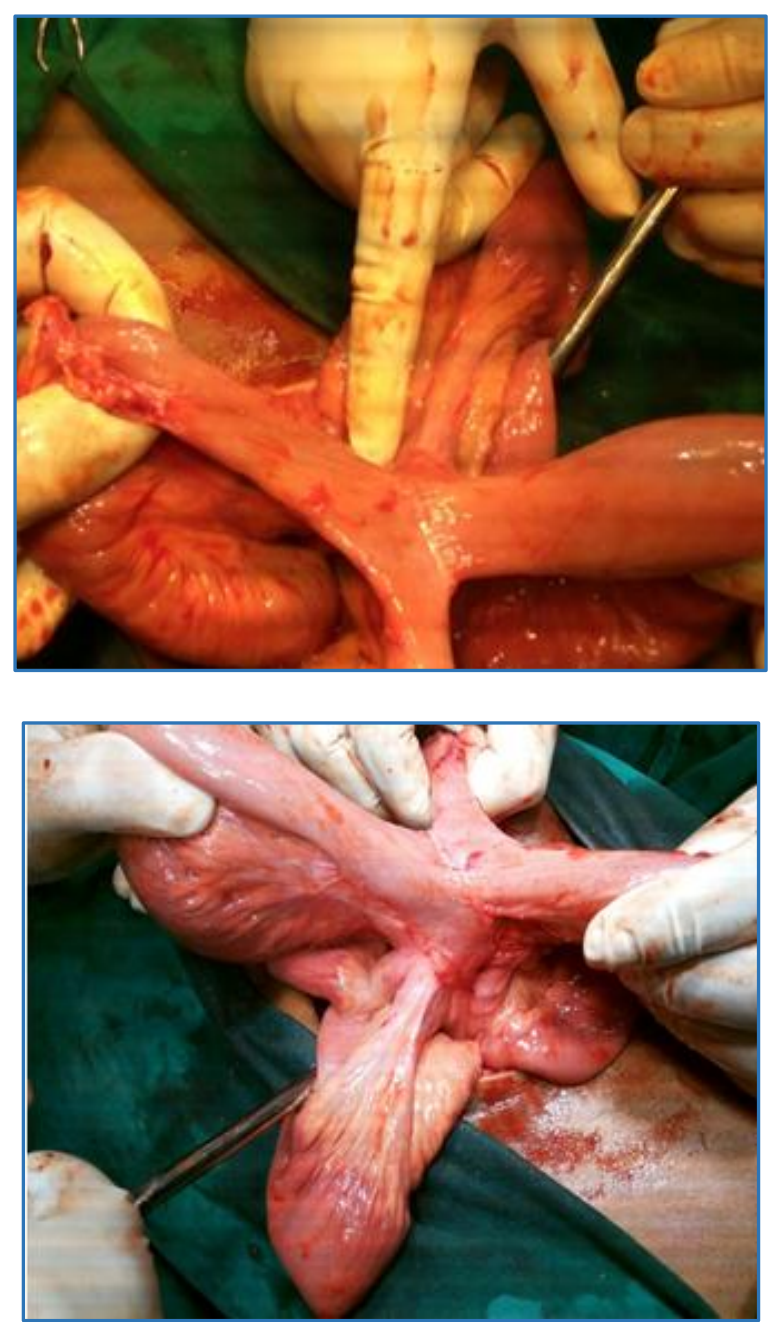

Figure 3 \& 4. Intraoperative Image showing Ileal Loops Adhered to Each Other Near Terminal Ileum

\section{Intraoperative Findings}

Band arising from the umbilicus is attached to the ileum. Ileal loops which are adhered to each other at the antimesenteric border was seen near terminal ileum, whereas jejunum was normal. Hence, planned for right hemicolectomy along with resection of diseased part of ileum.

\section{Gross Pictures}

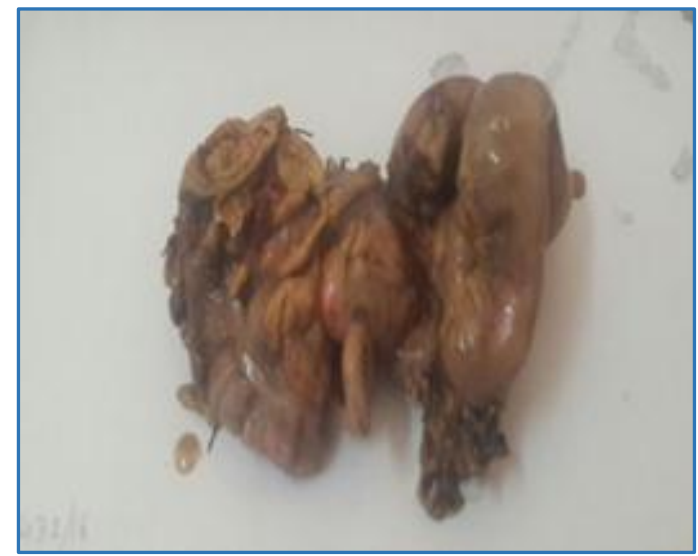

Figure 5. Specimen showing Features of Ileal Duplication

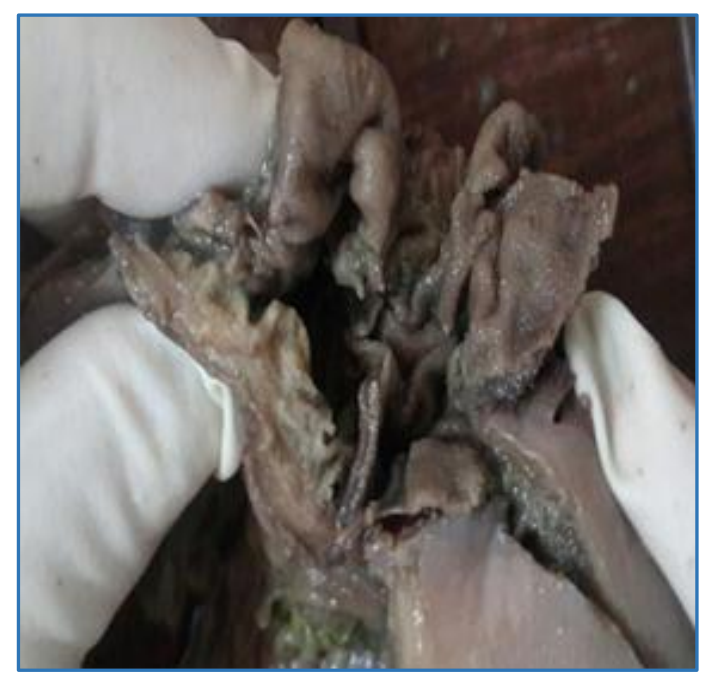

Figure 6. Cut Section showing Two Ileal Loops

\section{Histopathology Pictures}

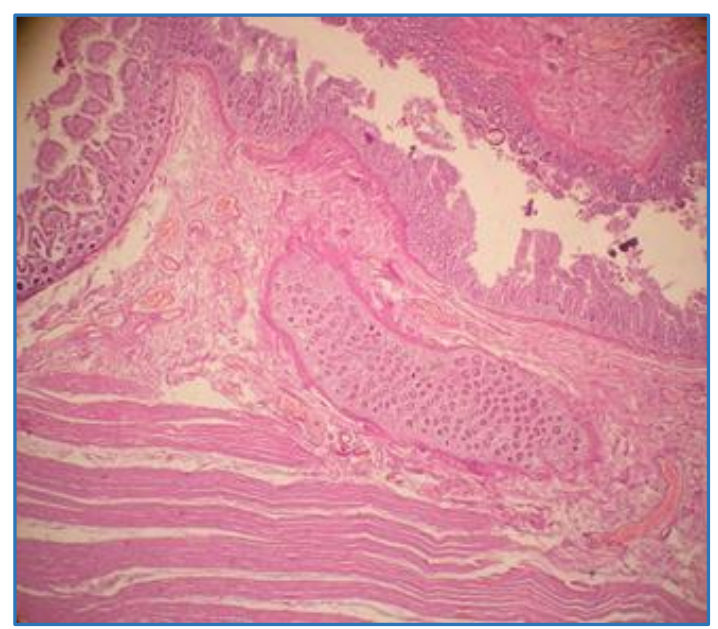




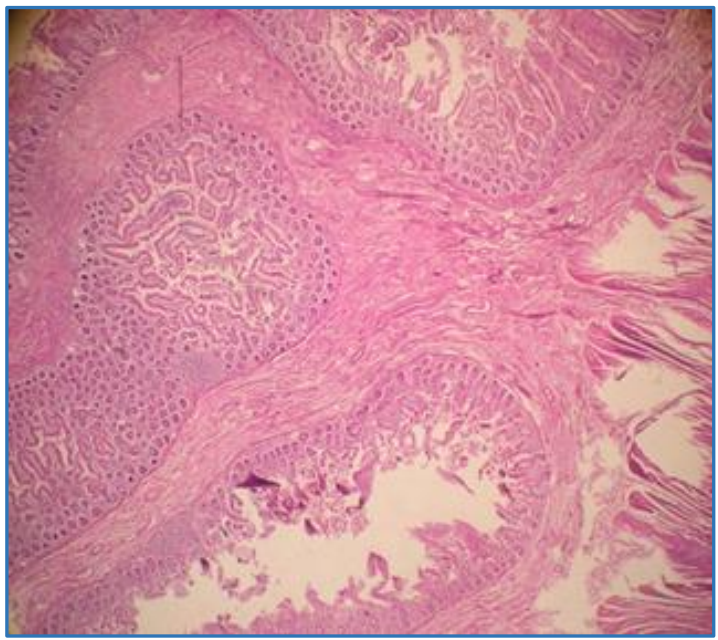

Figure 7 \& 8. Ileal Duplicate Lumen (H\&E) showing Gastric Heterotopic Tissue with Prominent Muscularis Layer

\section{Histopathology Reporting}

Specimen shows features of ileal duplication lined with gastric heterotopia.

Omentum shows features of congestion.

10 mesenteric lymph nodes: Reactive follicular hyperplasia with dilated sinuses.

\section{DISCUSSION}

Duplication of gastrointestinal system is a rare congenital malformation observed in one out of 25000 deliveries. This rare congenital anomaly was first described by Fitz in 1884, but was not widely used until it was popularised by Ladd in the 1930s.[4]

In 1937, Ladd defined "alimentary tract duplications" as congenital malformations that involve the mesenteric side of alimentary tract and share a common blood supply with the native bowel.[5]

It is typically found in children and only $20 \%$ of patients are diagnosed after 12 years of age.[6]

Ileal duplication can be classified according to the shape as cystic (noted in more than $80 \%$ ) or tubular.[7]

About $80 \%$ of intestinal duplications are found in the abdomen, $20 \%$ in the thoracic cavity. The supposed origin of alimentary tract duplication may be from the foregut, the midgut or the hindgut. The most commonly affected structures are ileum (35\%), oesophagus (19\%), jejunum (10\%), stomach $(9 \%)$ and colon $(7 \%) .{ }^{[8]}$

It is possible that duplication at different anatomical sites develops through different mechanisms. Several theories have been suggested to explain the cause of duplication. Of these, persistence of foetal gut diverticula, defect in recanalisation of the solid stage of primitive gut, partial twining and the split notochord theories are popular.[9]

Most of the adult intestinal duplications are asymptomatic and remain undiagnosed for years. Sometimes, they may result in serious morbidity or mortality if left untreated. Intestinal duplications may have an acute presentation or chronic complaints. Commonly present as nausea, vomiting, abdominal pain, constipation, bowel obstruction, volvulus, palpable mass and bloody stool.[4]

Duplications of the ileum represent the most common form of alimentary tract duplication and typically appears as non-communicating spherical cysts of varying sizes. When they take the form of long tubular segments, they tend to distend and cause extrinsic compression leading to obstructive symptoms. They can be easily mistaken for mesenteric or omental cysts; hence, to be clarified by identification of a mucosal rather than endothelial lining.

The mucosal lining within alimentary tract duplications does not necessarily correspond with the adjacent normal intestine and may display components of several different types of GI tract mucosa. Non-communicating duplications typically contain clear alkaline fluid, except in cases where gastric mucosa is present (25\%) and acidic fluid is observed. In addition, non-activated pancreatic enzymes may also be observed in cases of ectopic pancreatic tissue within the duplication lesion. ${ }^{[5]}$

However, ileal duplication cyst lined by ciliated columnar and squamous epithelium has been rarely reported in the literature.[10,11] In our case it was lined by normal intestinal columnar epithelium with ectopic gastric tissue.

Because small intestine is a particularly difficult part of the bowel to visualise with routine endoscopic investigation, imaging studies have an important role in establishing the diagnosis.

Ideal treatment of ileal duplication includes total excision, if not possible subtotal excision and/or internal derivation can be done.[12] The overall outcome of surgery is generally favourable.

\section{CONCLUSION}

Ileal duplication is one of the rare congenital abnormality involving the alimentary tract. However, our case is rare as it is noted in adulthood with tubular variety of ileal duplication. Most of the adult intestinal duplications are asymptomatic and remain undiagnosed for years. Sometimes, they may result in serious morbidity or mortality if left untreated. The overall outcome of surgery is generally favourable. In our patient also recovery was uneventful.

\section{REFERENCES}

1. Fernandez DC, Machicado J, Davogustto G. Gastrointestinal stromal tumor arising from a gastric duplication cyst. ACGCR 2016;3(3):175-7.

2. Diaz-Cano SJ, Rivera-Hueto F, Mesa-Navarro A. Double duplication in a nonrotational colon study of a case associated with mucinous adenoma. Pathology Research and Practice 1995;191(5):415-9.

3. Pandey A, Singh S, Gupta V, et al. Malrotation with midgut volvulus associated with perforated ileal duplication. Journal of Indian Association of Pediatric Surgeons 2013;18(4):155-7.

4. Shah A, Du J, Sun Y, et al. Dynamic change of intestinal duplication in an adult patient: a case report and literature review. Case Reports in Medicine Article ID 297585, 2012;2012:1-3.

5. Ildstad ST, Tollerud DJ, Weiss RG, et al. Duplications of the alimentary tract. Clinical characteristics, preferred treatment, and associated malformations. Annals of Surgery 1988;208(2):184-9.

6. Khoury T, Rivera L. Foregut duplication cysts: a report of two cases with emphasis on embryogenesis. World Journal of Gastroenterology 2011;17(1):130-4. 
7. Ho Y. Total colorectal and terminal ileal duplication presenting as intussusception and intestinal obstruction. World Journal of Gastroenterology 2012;18(43):6338-40.

8. Zheng J, Jing $\mathrm{H}$. Adenocarcinoma arising from a gastric duplication cyst. Surgical Oncology 2012;21(2):e97-101.

9. Stern LE, Wamer BW. Gastrointestinal duplications. Seminars in Pediatric Surgery 2000;9(3):135-40.

10. Kim KH, Choi SC, Kang DB, et al. A case of ileal duplication cyst lined by ciliated and squamous epithelium. Korean J Gastroenterol 2009;54(1):42-5.
11. Rodesch G, Dargent JL, Haller A, et al. An unusual presentation of a cystic duplication of the sigmoid colon entirely lined with squamous epithelium. Journal of Pediatric Surgery 2009;44(9):1831-4.

12. Kamak I, Ocal T, Senocak ME, et al. Alimentary tract duplications in children: report of 26 years experience. Turkish Journal of Pediatrics 2000;42(2):118-25. 